\title{
Molecular characterisation of the dominant UK methicillin-resistant Staphylococcus aureus strains, EMRSA-15 and EMRSA-16
}

\author{
P. C. L. MOORE and J. A. LINDSAY \\ Department of Infectious Diseases, St George's Hospital Medical School, Cranmer Terrace, London \\ SW17 ORE
}

\begin{abstract}
Epidemic methicillin-resistant Staphylococcus aureus types 15 and 16 (EMRSA-15 and EMRSA-16) are the dominant types of MRSA found in UK hospitals, but accurate designation of strains has been difficult. Restriction fragment length polymorphism (RFLP) profiles of seven core virulence genes were used to classify unambiguously isolates of MRSA from St George's Hospital into two groups corresponding to EMRSA15 and EMRSA-16. Variants of both EMRSA-15 and EMRSA-16 isolates occurred that had lost virulence genes encoded on mobile genetic elements. EMRSA-16 isolates had core gene profiles identical to a cluster of previously characterised MSSA (methicillinsensitive $S$. aureus) isolates from St George's Hospital, suggesting that they have arisen from this source, or that loss of the accessory genetic element encoding methicillin resistance is frequent. EMRSA-15 and EMRSA-16 strains were distinct from other MRSA strains previously identified in UK hospitals, and always carried a mobile genetic element encoding multiple superantigens. These results contribute to the understanding of the types of MRSA found in UK hospitals, how they vary and how they arose.
\end{abstract}

\section{Introduction}

Now that the sequencing of six Staphylococcus aureus genomes is nearly complete [1] (www.sanger.ac.uk, www.tigr.org, www.genome.ou.edu), it is possible to compare strains to see how they differ genetically. About $78 \%$ of genes are conserved between strains with some allelic variation, whereas $22 \%$ of genes are accessory, i.e., entirely absent in other strains [2]. The allelic variation in core backgrounds is relatively stable and can be used as an indicator of genetic relatedness between strains. In contrast, the accessory regions are often large stretches of DNA carrying virulence genes, known as genetic elements. Many are mobile and can be identified as bacteriophages, pathogenicity islands, integrated plasmids or transposons [3,4]. Some are known to transfer at high frequency and might do so in the hospital environment [3,5]. Therefore, S. aureus genomes can be regarded as having relatively stable core backgrounds, but vary significantly by the

Received 14 Jan. 2002; revised version accepted 22 Feb. 2002.

Corresponding author: Dr J. A. Lindsay (e-mail: jlindsay@ sghms.ac.uk). incorporation of a selection of accessory regions. This raises several interesting questions about how a strain or clone of $S$. aureus should be defined, particularly for epidemiological purposes.

Allelic variation in the relatively stable core backgrounds has been used to group isolates recently evolved from a common ancestor (clones). Enright et al. [6] and Day et al. [7] used multi-locus sequencing typing (MLST), the sequencing and comparison of seven housekeeping genes, for this purpose. They grouped isolates into related clusters and explored relationships between clusters. A previous report used a variation on this approach, comparing the sizes of ClaI fragments carrying seven core virulence genes among MSSA isolates from hospitals [5]. Instead of detecting every sequence change in each gene, this method identifies sequence polymorphisms at restriction endonuclease sites, re-arrangements, deletions, insertions or duplications in each gene and the surrounding region. The method allowed isolates to be grouped into clusters and relationships between clusters could be established.

Mobile accessory genetic elements can move frequently between isolates and, possibly, even within patients 
during infection $[5,8]$. This frequent movement explains some of the difficulties associated with the current epidemiological typing methods, pulsed-field gel electrophoresis (PFGE) and phage typing, in determining the relatedness of $S$. aureus strains. For example, a bacteriophage integrating into the chromosome will cause a PFGE band to 'disappear' and a new band to appear that is $45 \mathrm{~kb}$ larger [9]. This is defined as a two-band change. Alternatively, if the phage contains a single SmaI site, one PFGE band will be converted to two new bands with a combined increase in size (three-band change), and so on. These patterns can rapidly become difficult to interpret. Similarly, an integrated phage or pathogenicity island will carry 'phage immunity', preventing related phages from lysing the host bacterium and altering the phage typing pattern $[3,4]$.

Whereas PFGE and phage typing have value in showing whether isolates from an outbreak are related, they are not useful for classifying epidemiologically unrelated isolates. For example, EMRSA-15 is a major clonal group of $S$. aureus in UK hospitals, but $8 \%$ of isolates identified by phage typing as EMRSA-15 were probably unrelated to typical EMRSA-15 strains identified by PFGE profile [10]. Of 145 isolates assigned as EMRSA-15 by PFGE typing, 22 different PFGE patterns were identified, each with up to five band differences from the nearest profile. These isolates also showed variation in phage typing pattern, or the loss of a $15-\mathrm{kb}$ pathogenicity island carrying the gene for enterotoxin $\mathrm{C}(\mathrm{sec})$, or both.

The characterisation of EMRSA-15 and EMRSA-16 strains is important because their emergence in UK hospitals has coincided with a substantial increase in the incidence of MRSA infections over the last 5 years, from $1-2 \%$ to $40 \%$ of all $S$. aureus infections in UK hospitals [11]. It is estimated that EMRSA-15 and EMRSA-16 now account for $>95 \%$ of MRSA bacteraemias in the UK [11]. These two types of MRSA are particularly adapted to cause problems in hospitals compared with other MRSAs, but the basis for this is unknown. To address these issues accurate classification is necessary together with an estimate of the amount of variation within each clonal type.

In this study the RFLP method (Southern blotting) was used to investigate MRSA isolates at St George's Hospital, a 1200-bed teaching hospital in south London. The seven core gene profiles were used to classify the isolates unambiguously as either EMRSA15 or EMRSA-16. Variation in the accessory virulence gene carriage was detected within each of these clonal groups. Finally, by comparing these strains with less dominant epidemic UK MRSA strains, accessory genes associated with EMRSA-15 and EMRSA-16 were identified.

\section{Materials and methods}

\section{Isolates}

Forty-three MRSA isolates from different patients were obtained from the Department of Medical Microbiology at St George's Hospital, London, chosen to reflect the spectrum of hospital MRSA isolates. The isolates were from patients on a range of wards and comprised 26 isolates from blood cultures, 7 from sputum samples, 4 nasal isolates, 6 wound swab isolates, 1 MRSA groin screening swab isolate, 1 drain fluid isolate and 1 catheter urine isolate. Isolates were recovered from nutrient agar slopes at room temperature and transferred to storage at $-70^{\circ} \mathrm{C}$ on Microbank $^{\mathrm{TM}}$ beads (Pro-Lab diagnostics). Representative strains of EMRSA-1 to EMRSA-14 and EMRSA-17 [12] were kindly provided by the Staphylococcal Reference Laboratory, Laboratory for Hospital Infection, Central Public Health Laboratory, Colindale, London.

\section{Methicillin resistance testing}

Methicillin resistance determination was based on the modified Stokes method [13]. Briefly, test and control strains were grown on nutrient agar with salt $5 \%$ with a $10-\mu \mathrm{g}$ methicillin disk at $37^{\circ} \mathrm{C}$ for $18 \mathrm{~h}$, and a resistant strain had a zone of growth inhibition at least $3 \mathrm{~mm}$ smaller than the sensitive control. The presence of the $m e c A$ gene was confirmed by Southern blotting [5].

\section{Southern blotting}

Seven core genes were investigated: (i) the accessory gene regulator (agr) effector molecule, rnaIII; (ii) the regulator sarA; (iii) V8 serine protease, ssp; (iv) surface protein A, spa; (v) intercellular adhesin component, icaA; (vi) fibrinogen binding protein A, fnbA; and (vii) $\gamma$-haemolysin, hlg. A range of accessory virulence genes was also tested, including $\alpha$ - and $\beta$-haemolysins (hla, hlb), surface-binding proteins (cna, sbi, efb), capsule types 5 and 8 (cap5, cap 8$)$, heat-shock protein 70 ( $h s p 70)$, enterotoxins A-I (sea, seb, sec, sed, see, seg, seh, sei), toxic shock syndrome toxin-1 (tst), leukocidin E (lukE), staphylokinase (sak) and a serine protease-like protein, $s p l B$. The genes were chosen because they are known or potential virulence factors. Primers used to amplify each of these genes by PCR were as listed in an earlier report [5]. PCR products were labelled with horseradish peroxidase and hybridised to Southern blot membranes carrying ClaI-digested and electrophoresed whole genomic DNA from test isolates. Binding of the PCR probe was detected by enhanced chemiluminescence (Amersham). The size of the ClaI fragment carrying the gene in question was determined by comparing band sizes to a standard $\lambda$ DNA HindIII digest [5]. 


\section{Phage typing}

Isolates were kindly phage typed by the Staphylococcal Reference Laboratory.

\section{Results and discussion}

Two core gene profiles were identified, with 18 isolates showing one pattern and 25 isolates the other. Phage typing of the first 18 isolates identified 16 as EMRSA15 and the remaining two were non-typable. These 18 isolates have been called EMRSA-15. Of the 25 isolates with the second core gene profile, 24 were identified by phage typing as EMRSA-16, and one was non-typable. These 25 isolates have been called EMRSA-16. This is illustrated in Table 1, and the two core gene profiles are shown in Table 2. As only two patterns were identified, it is possible that the core genome approach is not very discriminatory. However, a previous study with MSSA [5] and the current study of EMRSA strains (see below) show that this is not the case. Rather the results reflect the dominance of these two clonal types. Therefore, it is proposed that these RFLP patterns could define EMRSA-15 and EMRSA16 clonal types. MLST would also provide a suitable definition $[6,7]$.

The core genomes of both EMRSA-15 and EMRSA-16 were highly conserved, but the accessory gene carriage within each of these two groups showed variation. This variation is highlighted in Table 1. Some EMRSA-15 isolates had lost sec and sak genes, which are carried on pathogenicity islands and phages, respectively. The variation in $s b i$ (IgG-binding protein) patterns could be due to the loss of a second copy of the sbi gene. As expected, $h l b$ showed variation because it is an integration site for bacteriophages, including phages carrying sak. Amongst the EMRSA-16 isolates, one had lost $t s t$, which is carried on a pathogenicity island. Capsule type (cap8) had been lost or altered in one isolate, which could account for its increased resistance to phage lysis (Table 1). PM79 had an altered hlb profile, possibly indicating that a phage that confers immunity to lysis by group III phages (including 75 and 77) had integrated into this gene. Therefore, variation in accessory gene carriage probably accounts for differences in phage profile or PFGE profile typically seen within each of these clonal types. Furthermore, loss of putative virulence genes, such as $s e c$ and $s a k$, from strains still able to cause infection might indicate that these genes have a limited role in infection. On the other hand, the loss of genes only from isolates not associated with disease, such as tst in a single nasal isolate, might support their importance in pathogenesis.

Notably, EMRSA-16 had an identical core genome profile to the MSSA agr class III cluster from a previous study (Table 2) [5]. Similar results have been reported previously [6]. Furthermore, the accessory gene carriage pattern of most EMRSA-16 isolates was identical to one of the MSSA isolates of $a g r$ class III, PM116, including the hlb RFLP (data not shown) [5]. This suggests that these isolates are very closely related. It could mean that EMRSA-16 emerged as a variant of an MSSA agr class III strain that acquired methicillin resistance. Methicillin resistance is due to carriage of the mec gene cluster on a genetic element called SCCmec [14], and the acquisition of SCCmec by an MSSA strain in vivo during treatment has been documented [8]. Alternatively, agr class III strains could be variants of EMRSA-16 that have lost $\mathrm{SCCmec}$. SCCmec is unstable in some isolates during storage in the laboratory [15; unpublished observations], so this might also occur in the hospital environment. It is also possible that movement of this element in and out of these strains occurs frequently. These results are also interesting in light of the ongoing discussion about whether MRSA are less virulent than MSSA. The data suggest that there are few differences between some strains of MRSA and MSSA.

Compared with other EMRSA in the UK, EMRSA-15 and EMRSA-16 are more successful at surviving, colonising or spreading in the hospital environment, and this could be due to their distinct core background or to the carriage of unique accessory genes. The core gene profiles of hospital EMRSA-15 and EMRSA-16 isolates were not only different from each other, but were clearly different from other EMRSA strains (Table 2). EMRSA-3, the third most frequently isolated MRSA in the UK, was also unique. EMRSA-1, -4, -7, -9 and -11 were closely related to each other. EMRSA $-2,-5,-6,-8,-10,-12,-13,-14$ and -17 were similarly related to each other.

Accessory gene carriage between EMRSA-15 and EMRSA-16 compared with the other EMRSAs also varied. The most striking feature was that EMRSA-15, -16 and -3 all carried seg and sei, whereas none of the other isolates did. These two genes form part of a genetic element containing several enterotoxin genes known as $e g c$, the enterotoxin nursery [16], that could play a role in virulence. Similarly, EMRSA-15 and EMRSA-16 did not carry $l u k E$ and $s p l B$, but the other EMRSA types did. The results are consistent with the previous report that isolates carrying egc are usually missing $l u k E$ and $s p l B$ [5]. Clearly, lukE and $s p l B$ are not essential for EMRSA strains to cause disease or become established in hospitals. EMRSA-15 and EMRSA-16 also had a unique $f n b A$ RFLP compared with other EMRSA types. All these factors might influence the dominance of EMRSA-15 and EMRSA16 in hospitals.

The results of this study resolve some of the confusion surrounding the types of MRSA found in UK hospitals. They are predominantly from two distinct lineages that are essentially conserved, and some are related to 
Table 1. EMRSA-15 and EMRSA-16 variants

\begin{tabular}{|c|c|c|c|c|c|c|c|c|c|}
\hline Strain no. & Source & tst & $\mathrm{sec}$ & sak & cap5 & cap 8 & $h l b$ & $s b i$ & Phage type \\
\hline \multicolumn{10}{|c|}{ EMRSA-15 } \\
\hline PM25 & Blood culture & - & 9.4 & 19 & 4 & - & $12+19$ & $6+14$ & 75 \\
\hline PM28 & Blood culture & - & 9.4 & 19 & 4 & - & $12+19$ & $6+14$ & 75 \\
\hline PM31 & Blood culture & - & - & 19 & 4 & - & $12+19$ & $6+14$ & 75 \\
\hline PM32 & Blood culture & - & $\begin{array}{cc}- & \\
\end{array}$ & 19 & 4 & - & $12+19$ & $6+14$ & 75 \\
\hline PM41 & Blood culture & - & - & 19 & 4 & - & $12+19$ & $6+14$ & 75 \\
\hline PM56 & Blood culture & - & $\begin{array}{c}- \\
-\end{array}$ & $\begin{array}{ll}- & \\
\end{array}$ & 4 & - & 4 & $6+14$ & 75 \\
\hline PM60 & Nose swab (screen) & - & $\overline{-}$ & 19 & 4 & - & $12+19$ & $6+14$ & 75 \\
\hline PM61 & Sputum & - & 9.4 & 19 & 4 & - & $12+19$ & $6+14$ & 75 \\
\hline PM62 & Sputum & - & 9.4 & 19 & 4 & - & $12+19$ & $6+14$ & 75 \\
\hline PM77 & Blood culture & - & 9.4 & 19 & 4 & - & $4+12$ & $6+14$ & 75 \\
\hline PM78 & Blood culture & - & 9.4 & 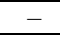 & 4 & - & 4 & 14 & 75 \\
\hline PM84 & Wound swab & - & 9.4 & 19 & 4 & - & $12+19$ & $6+14$ & 75 \\
\hline PM86 & Groin swab (screen) & - & 9.4 & 19 & 4 & - & $12+19$ & $6+14$ & 75 \\
\hline PM104 & Wound swab & - & - & 19 & 4 & - & $12+19$ & $6+14$ & 75 \\
\hline PM112 & Wound swab & - & 9.4 & 19 & 4 & - & $12+19$ & $6+14$ & 75 \\
\hline PM136 & Wound swab & - & 9.4 & 19 & 4 & - & $12+19$ & $6+14$ & Non-typable \\
\hline PM145 & Nose swab (screen) & - & 9.4 & 19 & 4 & - & $12+19$ & $6+14$ & Non-typable \\
\hline \multicolumn{10}{|c|}{ EMRSA-16 } \\
\hline PM5 & Sputum & 16 & - & 7.7 & - & $0.8+14$ & $6.7+7.7$ & 6 & $29,52,77$ \\
\hline PM6 & Sputum & 16 & - & 7.7 & - & $0.8+14$ & $6.7+7.7$ & 6 & $29,52,75,77,88 \mathrm{~A}, 932$ \\
\hline PM7 & Sputum & 16 & - & 7.7 & - & $0.8+14$ & $6.7+7.7$ & 6 & $29, \underline{52}, \underline{75}, \underline{77}$ \\
\hline PM11 & Blood culture & 16 & - & 7.7 & - & $0.8+14$ & $6.7+7.7$ & 6 & $29, \underline{52}, \underline{75}, \underline{77}$ \\
\hline PM17 & Blood culture & 16 & - & 7.7 & - & $0.8+14$ & $6.7+7.7$ & 6 & $29, \overline{52}, \underline{75}, \underline{77}$ \\
\hline PM22 & Sputum & 16 & - & 7.7 & - & $0.8+14$ & $6.7+7.7$ & 6 & $29,75,77,88 \mathrm{~A}, 932$ \\
\hline PM27 & Blood culture & 16 & - & 7.7 & - & $0.8+14$ & $6.7+7.7$ & 6 & $29, \underline{52}, \underline{75}, \underline{77}, \underline{83 \mathrm{~A}}$ \\
\hline PM30 & Blood culture & 16 & - & 7.7 & - & $0.8+14$ & $6.7+7.7$ & 6 & $29,52,7 \overline{79}, \underline{75}, \underline{77}, \underline{83 \mathrm{~A}}, \underline{83 \mathrm{C}}$ \\
\hline PM33 & Blood culture & 16 & - & 7.7 & - & $0.8+14$ & $6.7+7.7$ & 6 & $29, \underline{52}, \underline{75}, \underline{77}$ \\
\hline PM36 & Blood culture & 16 & - & 7.7 & - & $0.8+14$ & $6.7+7.7$ & 6 & $29, \underline{52}, \underline{75}, \underline{77}$ \\
\hline PM38 & Blood culture & 16 & - & 7.7 & - & $0.8+14$ & $6.7+7.7$ & 6 & $\underline{29}, \underline{52}, 52 \mathrm{~A}, \underline{79}, 80, \underline{75}, \underline{77}, \underline{83 \mathrm{~A}}, \underline{85}, \underline{83 \mathrm{C}}, \underline{932}, \underline{95}$ \\
\hline PM42 & Blood culture & 16 & - & 7.7 & - & $0.8+14$ & $6.7+7.7$ & 6 & $29, \underline{52}, \underline{75}, \underline{77}, \underline{83 \mathrm{~A}}, \underline{83 \mathrm{C}}$ \\
\hline PM53 & Blood culture & 16 & - & 7.7 & - & $0.8+14$ & $6.7+7.7$ & 6 & $\underline{29}, \underline{52}, 77,932, \underline{95}$ \\
\hline PM59 & Nose swab (screen) & - & - & 7.7 & - & $0.8+14$ & $6.7+7.7$ & 6 & $29, \underline{52}, \underline{75}, \underline{77}$ \\
\hline PM64 & Sputum & 16 & - & 7.7 & - & $0.8+14$ & $6.7+7.7$ & 6 & $29,52,75,77$ \\
\hline PM66 & Blood culture & 16 & - & 7.7 & - & $0.8+14$ & $6.7+7.7$ & 6 & $29,52,75,77,83 \mathrm{~A}, 83 \mathrm{C}$ \\
\hline PM67 & Blood culture & 16 & - & 7.7 & - & - & $6.7+7.7$ & 6 & Non-typable \\
\hline PM69 & Blood culture & 16 & - & 7.7 & - & $0.8+14$ & $6.7+7.7$ & 6 & 75 \\
\hline PM73 & Blood culture & 16 & - & 7.7 & - & $0.8+14$ & $6.7+7.7$ & 6 & $29,52,75,77,83 \mathrm{~A}, 83 \mathrm{C}$ \\
\hline PM79 & Blood culture & 16 & - & 7.7 & - & $0.8+14$ & $7.7+12$ & 6 & 29,52 \\
\hline PM85 & Wound swab & 16 & - & 7.7 & - & $0.8+14$ & $6.7+7.7$ & 6 & $29, \underline{52}, \underline{75}, \underline{77}, 83 \mathrm{C}$ \\
\hline PM87 & Drain fluid & 16 & - & 7.7 & - & $0.8+14$ & $6.7+7.7$ & 6 & $29, \underline{52}, \underline{75}, \underline{77}$ \\
\hline PM88 & Nose swab (screen) & 16 & - & 7.7 & - & $0.8+14$ & $6.7+7.7$ & 6 & $29,52,75,77,83 \mathrm{C}$ \\
\hline PM91 & Wound swab & 16 & - & 7.7 & - & $0.8+14$ & $6.7+7.7$ & 6 & $29,52,80, \underline{42 \mathrm{E}}, 47, \underline{77}, \underline{83 \mathrm{~A}}, \underline{83 \mathrm{C}}, \underline{81}$ \\
\hline PM92 & Catheter specimen of urine & 16 & - & 7.7 & - & $0.8+14$ & $6.7+7.7$ & 6 & $29, \underline{52}, \underline{75}, \underline{77}$ \\
\hline
\end{tabular}

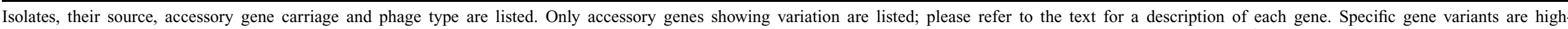

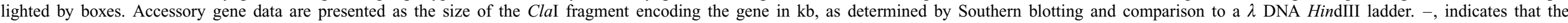

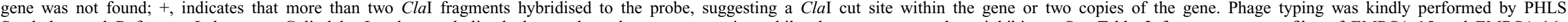

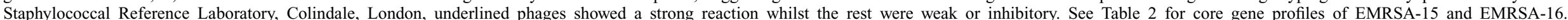


Table 2. Core gene and accessory gene profiles of MRSA

\begin{tabular}{|c|c|c|c|c|c|c|c|c|c|c|c|c|c|c|c|c|c|c|}
\hline \multirow[b]{2}{*}{ Strain type } & \multirow[b]{2}{*}{ Strain no. } & \multicolumn{7}{|c|}{ Core genes } & \multicolumn{10}{|c|}{ Accessory genes } \\
\hline & & $\begin{array}{c}\text { rnaIII } \\
\text { (agr class) }\end{array}$ & $\operatorname{sarA}$ & $s s p$ & spa & $i c a A$ & $f n b A$ & $h l g$ & $l u k E$ & $s p l B$ & seg & sei & tst & sak & sea & cna & cap 5 & cap8 \\
\hline EMRSA-15 & PM60 & $2.3+12$ (I) & 21 & 6.5 & 6.6 & 10 & 3.8 & $2.1+6+20$ & - & - & 8.2 & 8.2 & - & 19 & - & 6 & 4 & - \\
\hline EMRSA-16 & PM64 & $2.3+14$ (III) & 9.5 & $7+20$ & 7.9 & 10 & 3.8 & $2.1+6$ & - & - & 5.8 & 5.8 & 16 & 7.7 & $7.7+8.5$ & 17 & - & $0.8+14$ \\
\hline EMRSA-3 & M307 & $2.3+9$ (II) & 21 & 7 & 9.4 & 8 & 3.8 & $2.1+6$ & 3.8 & 9.4 & 5.3 & 4 & - & 9 & 9 & - & 4 & - \\
\hline $\begin{array}{l}\text { EMRSA-1 } \\
\text { EMRSA-4 } \\
\text { EMRSA-7 } \\
\text { EMRSA-9 } \\
\text { EMRSA-11 }\end{array}$ & $\begin{array}{l}\text { NCTC11939 } \\
\text { M211 } \\
\text { M063 } \\
\text { M203 } \\
\text { M695 }\end{array}$ & $\begin{array}{r}2+12 \text { (I) } \\
2.3+12 \text { (I) } \\
2.3+12 \text { (I) } \\
2.3+12 \text { (I) } \\
2.3+12 \text { (I) }\end{array}$ & $\begin{array}{l}21 \\
21 \\
21 \\
21 \\
21\end{array}$ & $\begin{array}{l}9 \\
9 \\
9 \\
9 \\
9\end{array}$ & $\begin{array}{l}6.6 \\
6.6 \\
6.6 \\
6.6 \\
6.6\end{array}$ & $\begin{array}{r}10 \\
10 \\
10 \\
10 \\
6\end{array}$ & $\begin{array}{l}2.8 \\
2.8 \\
2.8 \\
2.8 \\
2.8\end{array}$ & $\begin{array}{l}2.1+6 \\
2.1+6 \\
2.1+6 \\
2.1+6 \\
2.1+6\end{array}$ & $\begin{array}{l}24 \\
24 \\
24 \\
20 \\
24\end{array}$ & $\begin{array}{c}24 \\
24 \\
24 \\
8.9 \\
24\end{array}$ & $\begin{array}{l}- \\
- \\
- \\
- \\
-\end{array}$ & $\begin{array}{l}- \\
- \\
- \\
- \\
-\end{array}$ & $\begin{array}{l}- \\
- \\
- \\
- \\
-\end{array}$ & $\begin{array}{c}6.7 \\
6.7 \\
6.7 \\
19 \\
6.5\end{array}$ & $\begin{array}{c}2.5+6.7 \\
2.5+6.7 \\
2.5+6.7 \\
- \\
2.5+6.5\end{array}$ & $\begin{array}{l}9.4 \\
9.4 \\
9.4 \\
9.4 \\
9.4\end{array}$ & $\begin{array}{l}- \\
- \\
- \\
- \\
-\end{array}$ & $\begin{array}{l}0.8+14 \\
0.8+14 \\
0.8+14 \\
0.8+14 \\
0.8+14\end{array}$ \\
\hline $\begin{array}{l}\text { EMRSA-2 } \\
\text { EMRSA-5 } \\
\text { EMRSA-6 } \\
\text { EMRSA-8 } \\
\text { EMRSA-10 } \\
\text { EMRSA-12 } \\
\text { EMRSA-13 } \\
\text { EMRSA-14 } \\
\text { EMRSA-17 }\end{array}$ & $\begin{array}{l}\text { NCTC12232 } \\
\text { M527 } \\
\text { M406 } \\
\text { M597 } \\
\text { M103 } \\
\text { M258 } \\
\text { M274 } \\
\text { M585 } \\
\text { 99ST2758 }\end{array}$ & $\begin{array}{l}2.3+12 \text { (I) } \\
2.3+7.5 \text { (I) } \\
2.3+12 \text { (I) } \\
2.3+14 \text { (I) } \\
2.3+12 \text { (I) } \\
2.3+12 \text { (I) } \\
2.3+12 \text { (I) } \\
2.3+12 \text { (I) } \\
2.5+10 \text { (I) }\end{array}$ & $\begin{array}{l}21 \\
21 \\
21 \\
21 \\
21 \\
21 \\
21 \\
21 \\
21 \\
\end{array}$ & $\begin{array}{l}9 \\
9 \\
9 \\
9 \\
7 \\
9 \\
9 \\
9 \\
9\end{array}$ & $\begin{array}{l}9.4 \\
9.4 \\
9.4 \\
9.4 \\
9.4 \\
9.4 \\
9.4 \\
9.4 \\
9.4\end{array}$ & $\begin{array}{l}8 \\
8 \\
8 \\
8 \\
8 \\
8 \\
8 \\
8 \\
8\end{array}$ & $\begin{array}{l}2.8 \\
2.8 \\
2.8 \\
2.8 \\
2.8 \\
2.8 \\
2.8 \\
2.8 \\
2.8\end{array}$ & $\begin{array}{l}2.1+6 \\
2.1+6 \\
2.1+6 \\
2.1+6 \\
2.1+6 \\
2.1+6 \\
2.1+6 \\
2.1+6 \\
2.1+6\end{array}$ & $\begin{array}{l}6 \\
6 \\
6 \\
6 \\
6 \\
6 \\
6 \\
6 \\
5.5 \\
\end{array}$ & $\begin{array}{l}17 \\
17 \\
17 \\
17 \\
17 \\
17 \\
17 \\
17 \\
17\end{array}$ & $\begin{array}{l}- \\
- \\
- \\
- \\
- \\
- \\
- \\
- \\
-\end{array}$ & $\begin{array}{l}- \\
- \\
- \\
- \\
- \\
- \\
- \\
- \\
-\end{array}$ & $\begin{array}{l}- \\
- \\
- \\
- \\
- \\
- \\
- \\
- \\
-\end{array}$ & $\begin{array}{c}6.7 \\
6.7 \\
6.7 \\
23 \\
7.7 \\
19 \\
19 \\
19 \\
6.7\end{array}$ & $\begin{array}{c}2.5+6.7 \\
2.5+6.7 \\
2.5+6.7 \\
\quad- \\
7.7+8.5 \\
\quad- \\
- \\
- \\
7.7+8.5\end{array}$ & $\begin{array}{l}- \\
- \\
- \\
- \\
- \\
- \\
- \\
- \\
-\end{array}$ & $\begin{array}{l}4 \\
4 \\
4 \\
4 \\
4 \\
4 \\
4 \\
4 \\
4\end{array}$ & $\begin{array}{l}- \\
- \\
- \\
- \\
- \\
- \\
- \\
- \\
-\end{array}$ \\
\hline MSSAagr III & PM116 & $2.3+14$ (III) & 9.5 & $7+20$ & 7.9 & 10 & 3.8 & $2.1+6$ & - & - & 5.8 & 5.8 & 16 & ND & $7.7+8.5$ & 17 & - & $0.8+14$ \\
\hline
\end{tabular}

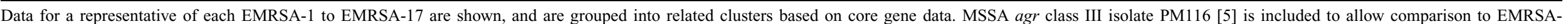

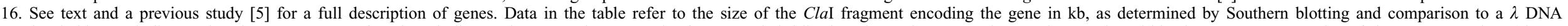

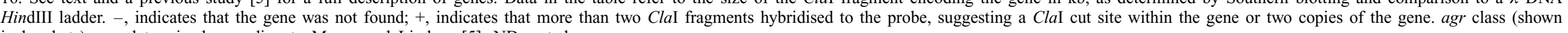
in brackets) was determined according to Moore and Lindsay [5]. ND, not done. 
MSSA found in hospitals. There is significant variation between related strains in their carriage of accessory genetic elements encoding virulence genes. The carriage of these genes might impact on the virulence of an isolate or its ability to survive in the hospital environment.

This work was funded by a Wellcome Trust Entry Level Fellowship 058051 to P.C.L.M. We thank the PHLS Staphylococcal Reference Laboratory, Colindale, for supplying reference strains of EMRSA and performing phage typing, and the Sanger Centre, TIGR and the University of Oklahoma for providing $S$. aureus sequence information before publication.

\section{References}

1. Kuroda M, Ohta T, Uchiyama I et al. Whole genome sequencing of methicillin-resistant Staphylococcus aureus. Lancet 2001; 357: 1225-1240.

2. Fitzgerald JR, Sturdevant DE, Mackie SM, Gill SR, Musser JM. Evolutionary genomics of Staphylococcus aureus: insights into the origin of methicillin-resistant strains and the toxic shock syndrome epidemic. Proc Natl Acad Sci USA 2001; 98: $8821-8826$.

3. Betley MJ, Mekalanos JJ. Staphylococcal enterotoxin A is encoded by phage. Science 1985; 229: 185-187.

4. Lindsay JA, Ruzin A, Ross HF, Kurepina N, Novick RP. The gene for toxic shock toxin is carried by a family of mobile pathogenicity islands in Staphylococcus aureus. Mol Microbiol 1998; 29: 527-543.

5. Moore PCL, Lindsay JA. Genetic variation among hospital isolates of methicillin-sensitive Staphylococcus aureus: evidence for horizontal transfer of virulence genes. $J$ Clin Microbiol 2001; 39: 2760-2767.

6. Enright MC, Day NPJ, Davies CE, Peacock SJ, Spratt BG.
Multilocus sequence typing for characterization of methicillinresistant and methicillin-susceptible clones of Staphylococcus aureus. J Clin Microbiol 2000; 38: 1008-1015.

7. Day NPJ, Moore CE, Enright MC et al. A link between virulence and ecological abundance in natural populations of Staphylococcus aureus. Science 2001; 292: 114-116.

8. Wielders CLC, Vriens MR, Brisse $\mathrm{S}$ et al. Evidence for invivo transfer of mecA DNA between strains of Staphylococcus aureus. Lancet 2001; 357: 1674-1675.

9. Smeltzer MS, Hart ME, Iandolo JJ. The effect of lysogeny on the genomic organization of Staphylococcus aureus. Gene 1994; 138: $51-57$.

10. O'Neill GL, Murchan S, Gil-Setas A, Aucken HM. Identification and characterization of phage variants of a strain of epidemic methicillin-resistant Staphylococcus aureus (EMRSA15). J Clin Microbiol 2001; 39: 1540-1548.

11. Johnson AP, Aucken HM, Cavendish S et al. Dominance of EMRSA-15 and -16 among MRSA causing nosocomial bacteraemia in the UK: analysis of isolates from the European Antimicrobial Resistance Surveillance System (EARSS). $J$ Antimicrob Chemother 2001; 48: 143-144.

12. Ayliffe GA, Buckles A, Casewell MW et al. Revised guidelines for the control of methicillin-resistant Staphylococcus aureus infection in hospitals. J Hosp Infect 1998; 39: 253-290.

13. Working Party of the British Society for Antimicrobial Chemotherapy. A guide to sensitivity testing. J Antimicrob Chemother 1991; 27 Suppl D: 1-48.

14. Katayama Y, Ito T, Hiramatsu K. A new class of genetic element, staphylococcus cassette chromosome mec, encodes methicillin resistance in Staphylococcus aureus. Antimicrob Agents Chemother 2000; 44: 1549-1555.

15. Inglis B, El-Adhami W, Stewart PR. Methicillin-sensitive and -resistant homologues of Staphylococcus aureus occur together among clinical isolates. J Infect Dis 1993; 167: 323-328.

16. Jarraud S, Peyrat MA, Lim A et al. egc, a highly prevalent operon of enterotoxin gene, forms a putative nursery of superantigens in Staphylococcus aureus. J Immunol 2001; 166: 669-677. 